\title{
Improving Concrete Containment Structures Associated with Fixed-Cone Valves
}

\author{
Bret Skyler Buck, Michael Clyde Johnson, Zachary Brad Sharp \\ Utah Water Research Laboratory (UWRL), Utah State University, Logan USA \\ E-mail: skyler.buck@aggiemail.usu.edu,michael.johnson@usu.edu, zac.sharp@aggiemail.usu.edu \\ Received March 3, 2011; revised March 29, 2011; accepted March 31, 2011
}

\begin{abstract}
Fixed-Cone valves are often used to dissipate energy and regulate flow at the low level outlet works of dams. Fixed-Cone valves, also known as Howell-Bunger valves, create an expanding conical jet allowing the energy of the water to dissipate over a large area. However, in many applications constructing the large stilling basin necessary for these valves is either not possible or not feasible. In order to reduce the relative size of the stilling basin, hoods or concrete containment structures have been used in conjunction with Fixed-Cone valves. This paper discusses the use of baffles in concrete containment structures in order to dissipate energy in a considerably confined space. It was determined that using baffles, in place of a deflector ring and end sill (Used in traditional containment structures), significantly improves the function of containment structures by reducing downstream flow velocities and improving flow patterns and stability. This information will be useful to engineers allowing them to minimize scour and erosion associated with concrete containment structures.
\end{abstract}

Keywords: Valves, Containment Structure, Energy Dissipation, Concrete Erosion, Outlet Works

\section{Introduction}

The Howell-Bunger valve, also known as the FixedCone valve, is often used to reduce energy in the water exiting the low level outlet works of a dam. This reduction of energy must happen in order to avoid erosion at the toe of dam or in downstream channels. It is especially important to reduce the velocity in the downstream channel because velocity is the primary source of erosion and scour.

Originally introduced by C. H. Howell and H. P. Bunger in 1935, the valve consists of a conical Section that is fixed in the end of the valve with a telescoping sleeve that regulates flow. The valve causes the water exiting to expand out radially creating a conical spray. It is common for the water exiting the valve to exit at either 45 or 30 degrees measured from an axis that extends perpendicular from the pipe. These valves are commonly used to dissipate energy and regulate flow from the outlet works of dams with medium (35-165 ft) to high $(>165$ $\mathrm{ft}$ ) heads. The Fixed-Cone valve is not only an excellent energy dissipater, it is also is an excellent way to aerate water discharged from impoundments. This is primarily due to the fact that the water exiting a Fixed-Cone valve expands out in every direction thus allowing a large flow surface to be in contact with the atmosphere [1].

When used alone Fixed-Cone valves dissipate energy effectively, however, due to the expanding conical jet, relatively large stilling basins are required to capture the excessive overspray. In many applications a large stilling basin is either not possible or not feasible. In order to reduce the size of the stilling basin, hoods and concrete containment structures have been used in conjunction with Fixed-Cone valves. In applications with medium heads, hoods are often used in conjunction with FixedCone valves creating a concentrated hollow jet. When the hood is attached to the valve it is referred to as a Ring-Jet valve. However, Ring-Jet valves and Hooded Fixed-Cone valves still require a considerable sized stilling basin in order to avoid having a scouring effect take place downstream. In order to dissipate more energy the hoods can be lined with baffles. The combination of a Fixed-Cone valve and a baffled hood are capable of dissipating up to 95 percent of the power upstream from the valve [2].

The United States Bureau of Reclamation (USBR) has several designs using Howell-Bunger valves in conjunction with reinforced concrete containment structures. The 
containment structures vary in size and cross- sectional shape, but maintain the same general design and similar structural elements. These containment structures usually include an aeration hatch, a Fixed-Cone valve, and a deflector ring followed by an end sill or baffle piers. When in operation this valve produces a conical jet that strikes the walls of the containment structure at approximately 45 degree angles (only if a 90 degree cone is used). After contact most of the flow continues along the surface until the deflector ring redirects the flow to a common point downstream [3].

The USBR has employed these concrete containment structures at a number of dams including: LG-2 Development (Quebec, Canada), Portage Mountain Dam (British Columbia, Canada), Ute Dam (New Mexico, USA), New Waddell Dam (Arizona, USA), Stony Gorge Dam (California, USA), and Jordanelle Dam (Utah, USA). It has been noted that the structure at Jordanelle Dam has a considerable amount of overspray even at low flows. The concrete containment structure at the LG-2 development consists of two Fixed-Cone Valves discharging into a common chamber of oval cross-Section with a deflector ring followed by a row of floor baffles [4]. The Portage Mountain Dam structure has a circular cross-Section, but in all other regards is the same as the LG-2 structure [5]. The Ute dam has a chamber cross-Section that is octagonal, followed by the deflector ring and an end sill instead of the floor baffles [6]. The other dams listed have containment structures with rectangular cross-Sections deflector rings and end sills [4].

\section{Experiments}

A study was conducted at Utah State University at the Utah Water Research Laboratory (UWRL) to determine if there was a more effective and economical containment structure that could be used with Fixed-Cone valves. A fixed cone valve having 7.8-inch fixed cone diameter and an exit angle of 45 degrees was used for these tests. Six different models were constructed and compared for this study. Figure 1 shows the two different containment structure cross-sections (with their respective dimensions) used for this research, with the dimensions standardized in terms of valve diameter (D). Each cross-Section had three configurations that were tested. The first configuration used a deflector ring and an end sill. Figure 2 shows the profile and plan views of the standard containment structure configuration described previously with deflector ring and end sill. The other two configurations used the baffles shown in Figure 3 instead of the deflector ring and end sill, the only difference being that the last two rows of baffles shown in Figure 4 were removed for the third configuration.
Once again, note that all dimensions were normalized in terms of the valve diameter in order to easily apply them to any desired prototype. Plywood painted with a latex paint was the construction material used to simulate the concrete containment structures, the deflector ring and the end sill while Plexiglas was used to make the baffles. The six models were run through four different model reservoir heads with five different flow rates for each reservoir head. The model reservoir heads for this experiment were 15.4D, 23.1D, 30.8D, and 38.5D.
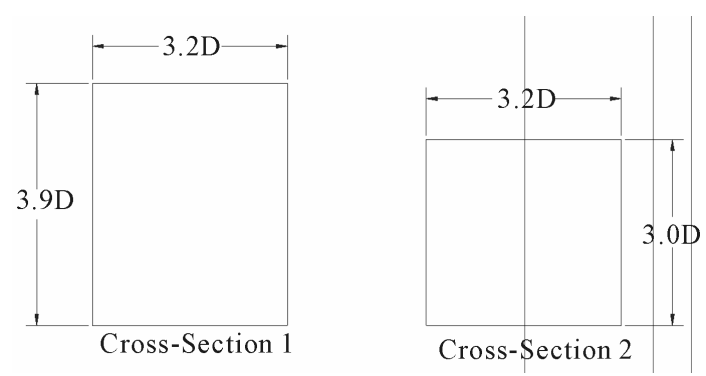

Figure 1. Cross-sections of containment structures.
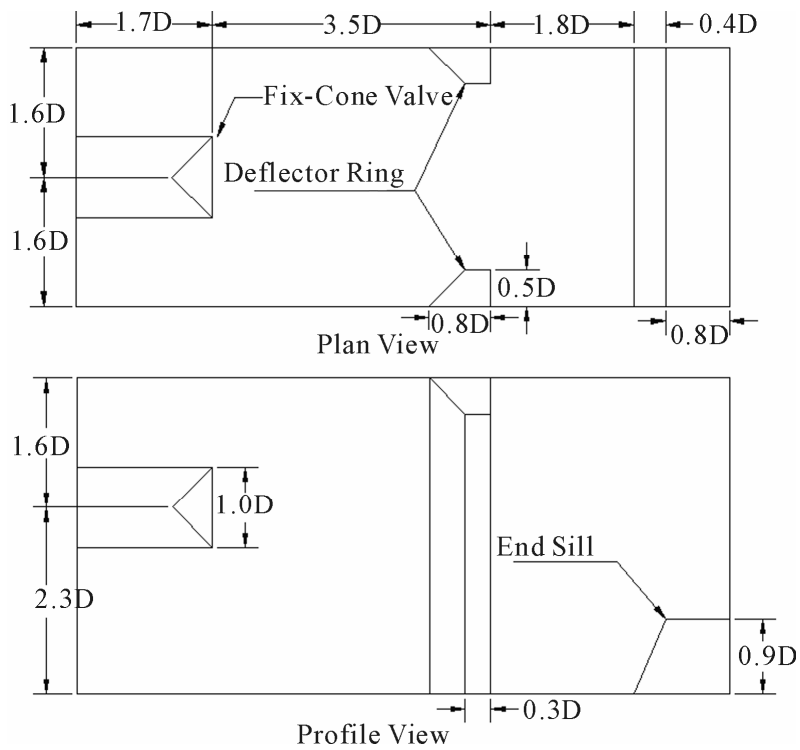

Figure 2. Containment structure with deflector ring and end sill in cross-Section 1.

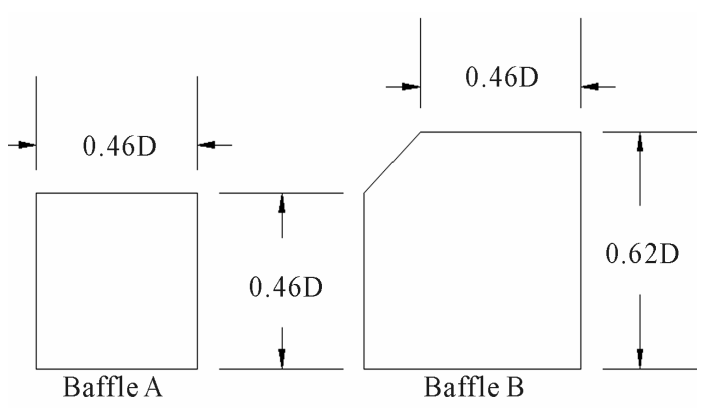

Figure 3. Standard baffle A and corner baffle B. 


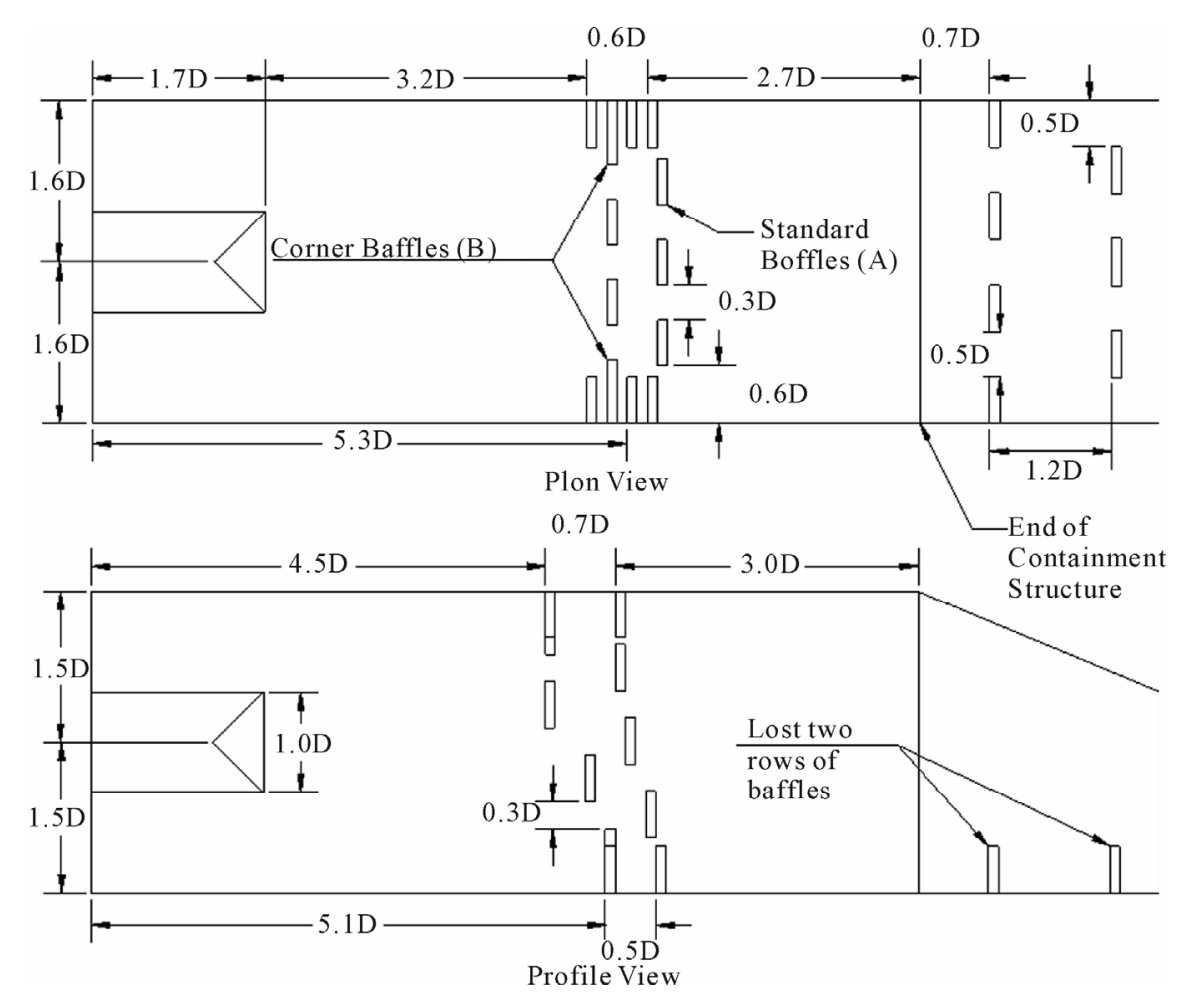

Figure 4. Baffle containment structure with extra two rows of baffles in cross-section 2.

\section{Evaluation Criteria}

All previous research and model studies focused solely on adequate energy dissipation and observations. These are both valid and important criteria and are hence included in this study. However, in order to more thoroughly evaluate the performance of the model containment structures downstream velocities and the downstream flow patterns were also taken into account. These two criterions are of utmost importance. Undesirable flow patterns, including non-uniform flow and unstable hydraulic jumps, require additional design considerations for the downstream channel. These considerations could represent a considerable cost both in time and structures to ameliorate the channel. High velocity flows are of highest significance when considering scour and erosion of material. In this study average velocities in the downstream channel were used for comparison. In order to calculate these velocities the flows were divided by the product of the average depth of flow and the width of the downstream channel. The flow patterns were qualitatively recorded by noting any hydraulic jumps, flow patterns or irregularities.

The amount of energy dissipated $\left(E_{d}\right)$ is calculated using Equation (1), and is given as a percentage of the initial energy head $\left(H_{i}\right)$ minus the final energy head $\left(H_{f}\right)$ over the initial head.

$$
E_{d}=\left(\frac{H_{i}-H_{f}}{H_{i}}\right) * 100
$$

$H_{i}$ and $H_{f}$ are calculated using Equation (2) where the energy head or total head $(\mathrm{H})$ is calculated employing the following form of the Bernoulli Equation:

$$
H=\frac{P}{\gamma}+Z+\frac{V^{2}}{2 g}
$$

where $\mathrm{H}$ is the total head, $\mathrm{P}$ is the pipe pressure at the inlet of the Howell-Bunger valve, $\gamma$ is the specific weight of water, $\mathrm{Z}$ is the elevation of the water above datum, $\mathrm{g}$ is the acceleration due to gravity, and $\mathrm{V}$ is the average velocity. The elevation datum for the water was taken as the bottom of the containment structure. The initial head was calculated from measurements made upstream of the Howell-Bunger valve using a precision pressure gage to measure the pipe pressure and a calibrated magnetic flow meter to measure the flow rate. The final head was calculated using the same flow rate found upstream of the Howell-Bunger valve and a channel depth. The downstream water depth was taken as the average of three depths (at $0.25,0.5$, and 0.75 the width of the channel) which were measured using a point gauge downstream from the containment structure. Measurements were also taken for the geometry of the different containment structures (cross-Section 1 or cross-Section 2), the per- 
cent valve opening, and the velocity of air entering though the aeration hatch.

\section{Results}

It was originally planned to record a baseline dissipation measurement using cross-Section 1 without any of the energy dissipation structures installed. However, the water exiting the structure was moving at a high rate of velocity and was far too turbulent to get any valid readings. This visually confirmed the fact that simply changing the direction of the jet from a Fixed-Cone valve does little as far as power (energy) dissipation is concerned [2].

The two models with four rows of floor baffles allowed for the greatest uniformity and stability in the flow pattern in the downstream channel. The configurations with the end sills always had a hydraulic jump in the channel though the location changed based on flow, and even then the jumps tended to shift locations. The hydraulic jumps formed because as flow exits the structure it is accelerated off the end sill. Figure 5 shows photographs of these two configurations with the same model head of $38.5 \mathrm{D}$ and the highest flow tested. The hydraulic jump can be seen on the left, but it should be noted that its location did shift while the baffles maintained a uniform flow pattern. The flow patterns observed are representative of both structures throughout the range of flows and heads. The deflector ring with end sill configurations also exhibited a strange V-shaped flow pattern where the depth in the center of the channel was noticeably lower and the velocity noticeably higher than at the sides of the channel. This design also had a lot more overspray at the end of the channel when compared to the baffle designs.

For plotting and comparison purposes dimensionless terms were used, including a theoretical jet Froude number. The theoretical Froude number is calculated assuming that all the head at the valve, excluding the elevation head, converts to velocity head. This assumption is
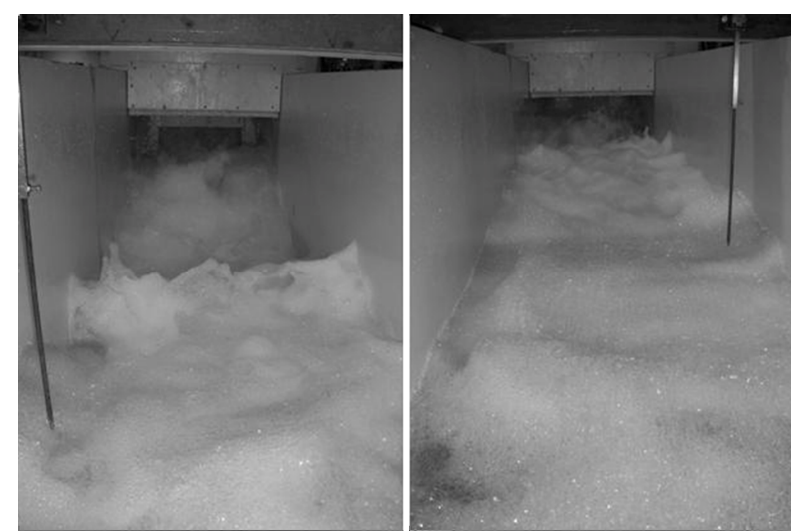

Figure 5. Comparison of deflector ring with end sill flow (left) to baffle flow with 4 rows of floor baffles (right). made because the containment structures are vented to the atmosphere therefore the water pressure as the water jet leaves the valve is zero. The Froude number of the jet, $F_{j}$, is calculated using Equation (3) where $h$ is the summation of the pressure head and the velocity head upstream from the valve, $g$ is the acceleration due to gravity and $t$ is the thickness of the jet.

$$
F_{j}=\frac{\sqrt{2 g h}}{\sqrt{g t}}
$$

The thickness of the jet, $t$, was computed using Equations (4) and (5):

$$
\begin{gathered}
A_{j}=\frac{Q}{\sqrt{2 g h}} \\
t=\sqrt{\frac{A_{j}}{\pi}+R^{2}}-R
\end{gathered}
$$

where $Q$ is the flow going through the valve, $A_{j}$ is the theoretical area of the jet, $\mathrm{R}$ is the radius of the cone at the outlet and all other variables are as previously defined.

Figure 6 shows the percent of energy dissipated plotted against the theoretical Froude number of the flow exiting the Fixed-Cone valve. It is noteworthy that the larger Froude numbers correspond to low flows with small valve openings, while the lower Froude numbers are higher flows with larger valve openings. It is apparent that at low flows all the containment structures performed similarly and that only at medium to high flows was there a measureable difference in energy dissipation. The design with the deflector ring and the end sill with a larger cross-sectional area and the baffle design with the extra two rows of baffles had no definitive energy dissipation differences. These designs always had energy dissipation measurements within one percent of one another. Larger floor baffles could increase the amount of drag on the water leading to greater energy dissipation; however the baffles more than adequately reduced the velocities in the downstream channel which is most important and it was therefore determined that further experimentation with the size and location of baffles was not needed at this time.

To compare the downstream velocities of the models, the downstream velocity was divided by the theoretical velocity of the jet exiting the Howell-Bunger valve in order to get a dimensionless quantity. Figure 7 displays this dimensionless velocity number plotted versus the theoretical Froude number. As the figure shows, the relative downstream velocities associated with the baffle configurations that have the extra two rows of floor baffles produced drastically lower velocities exiting the chamber. The higher velocity flows always initiated a hydraulic jump in the channel which often shifted loca- 
tion. The high velocities as well as a rapidly shifting hydraulic jump would require greater engineering considerations to avoid hydraulic damage in the form of scour or up-lift of concrete in the downstream channel. For these reasons and the fact that there was not a pronounced difference in energy dissipation, the baffle configuration with the extra two rows of baffles was the preferred configuration.
Figure 8 displays air flow demands of the structures, the ratio of air flow to water flow is plotted against the theoretical Froude number. It is interesting to note that the smaller cross-section had a smaller ratio of air flow to water flow. This could result less effective aeration of the water flowing through the structure, but more likely this is simply a result of less air being evacuated from the chamber with the water.

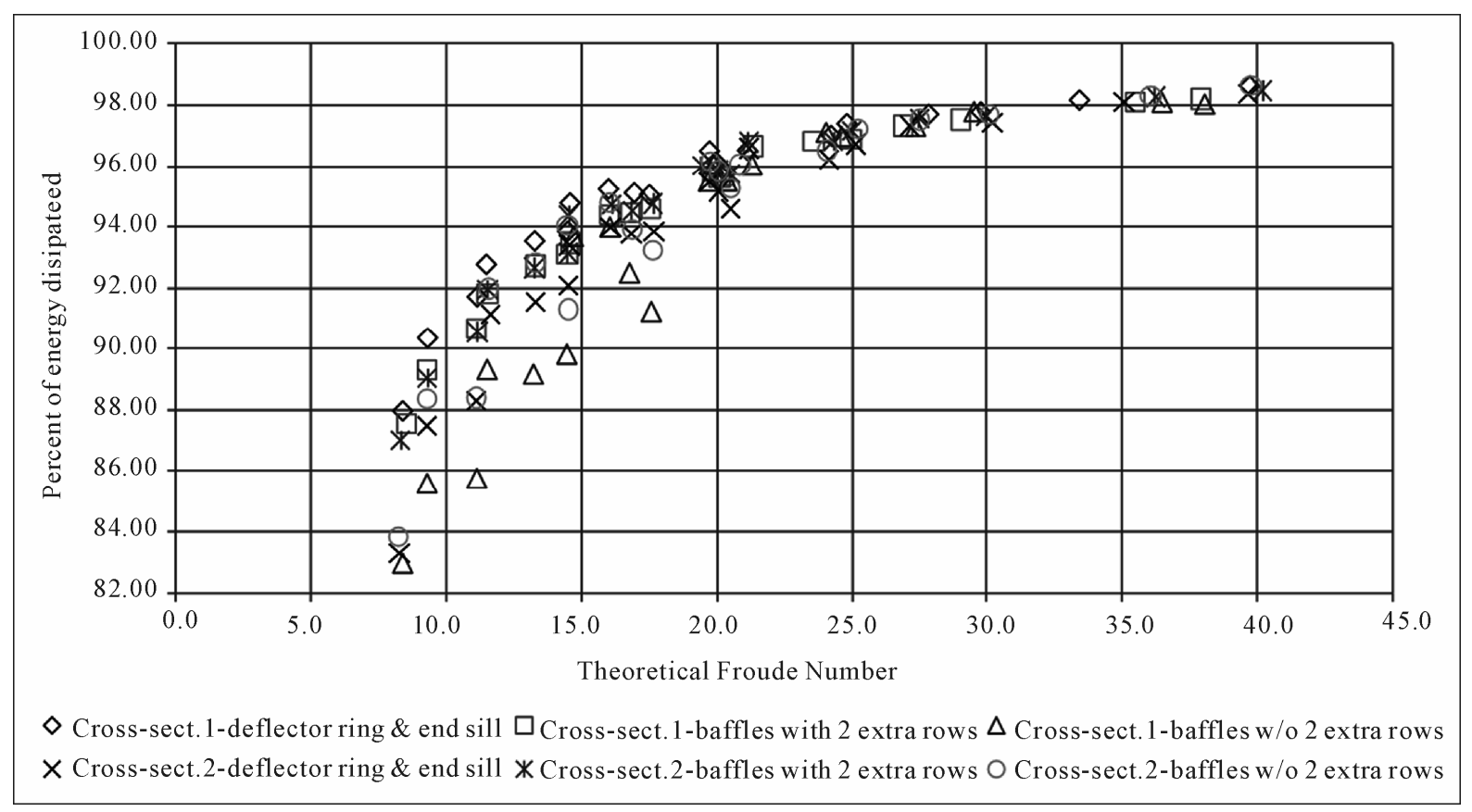

Figure 6. Energy dissipation versus theoretical Froude number.

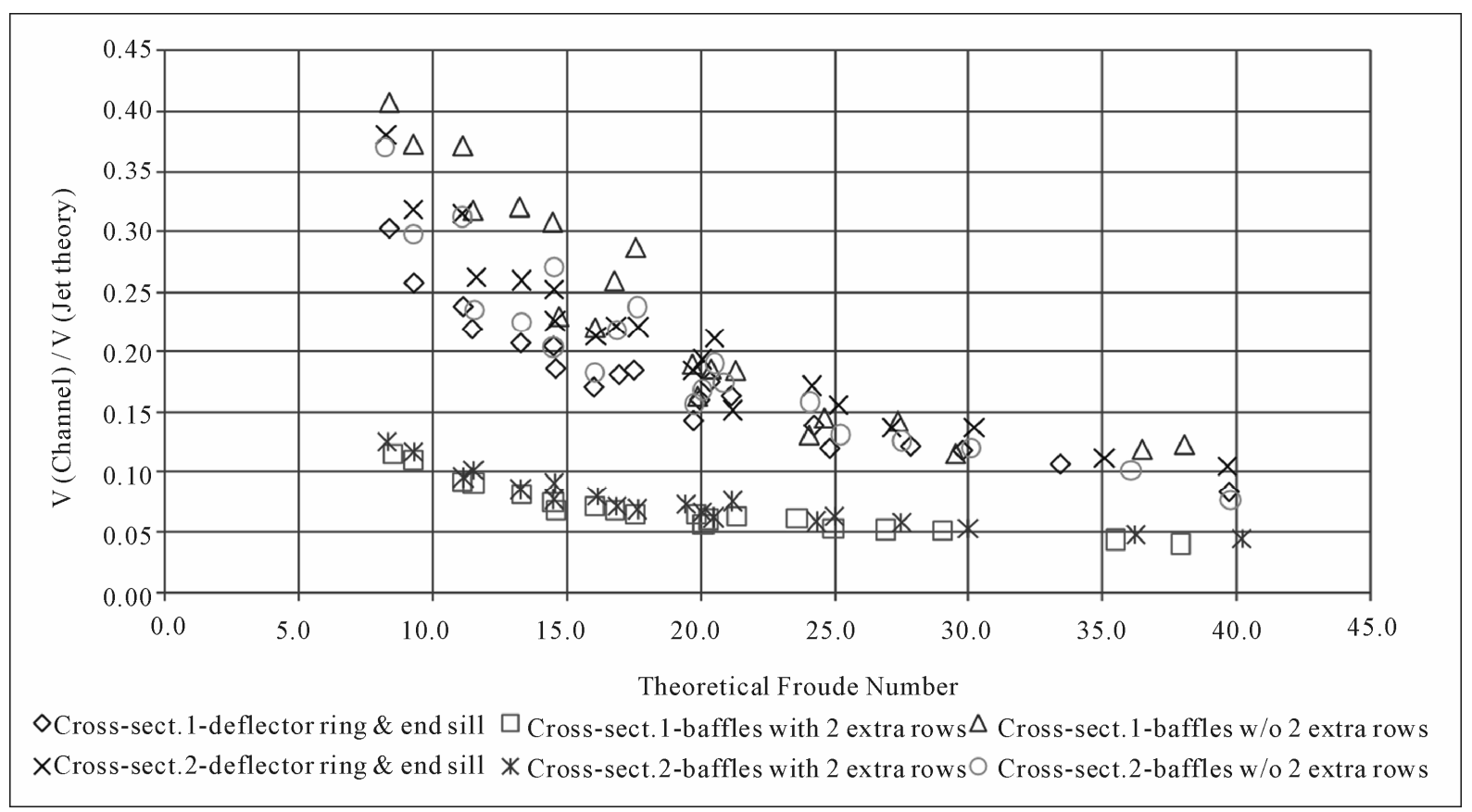

Figure 7. Ratio of downstream velocity/theoretical velocity versus theoretical Froude number. 


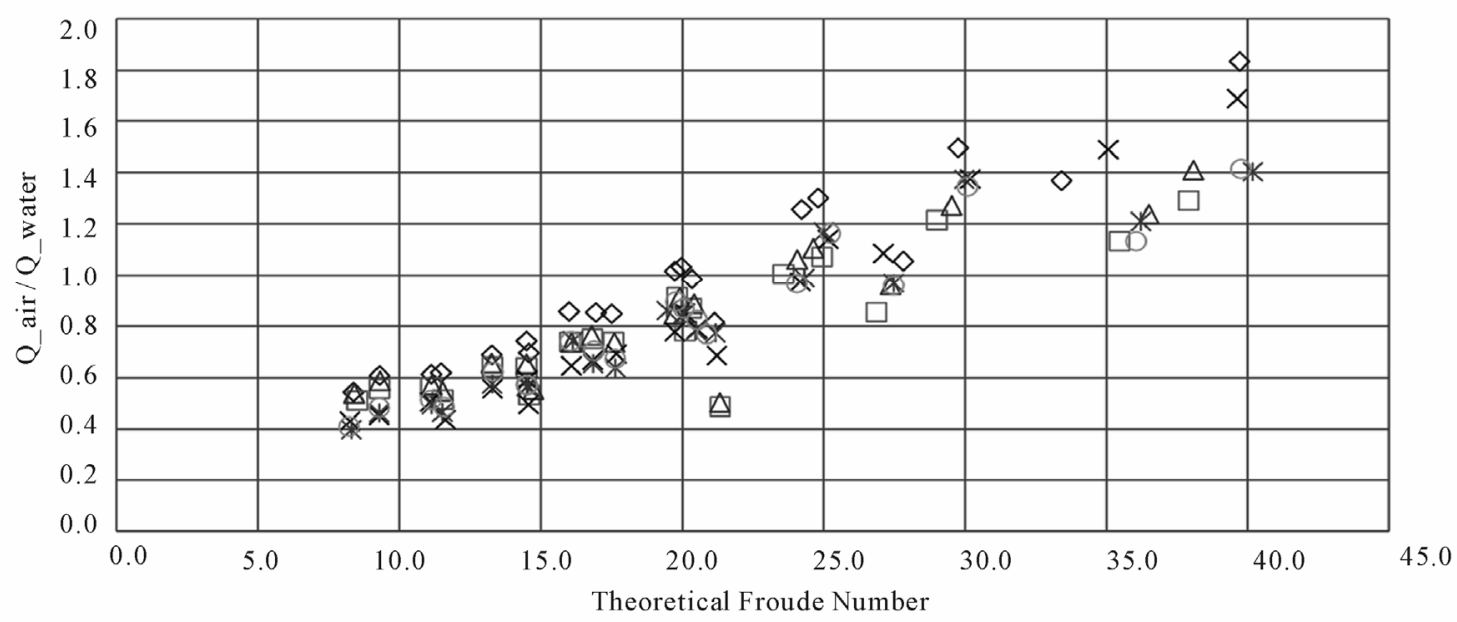

$\diamond$ Cross-sect. 1-deflector ring $\&$ end sill $\square$ Cross-sect.1-baffles with 2 extra rows $\triangle$ Cross-sect. 1-baffles w/o 2 extra row s $\times$ Cross-sect. 2 -deflector ring $\&$ end sill $*$ Cross-sect.2-baffles with 2 extra rows $\bigcirc$ Cross-sect. 2 -baffles w/o 2 extra row s

Figure 8. Ratio air flow/water flow versus theoretical Froude number.

\section{Discussion}

This study was not an exhaustive study in order to discover the single most efficient containment structure that could be used in conjunction with Fixed-Cone valves, but rather an investigation of baffled teeth energy dissipation with earlier energy dissipation methods. Using teeth like baffles for energy dissipation in concrete containment structures is a novel idea and there is little research in this area, however these teeth like projections have been used in conjunction with Fixed-Cone valve hoods and successfully dissipated energy in that application with the introduction of the baffled-hood [2].

Though energy dissipation has been the primary indicator of the effectiveness of these structures in previous experiments, it became apparent that the downstream water velocities and flow patterns are of even greater importance. Most energy dissipation structures at the low level outlet works are built to minimize scour and damage downstream, while energy dissipation is simply a measure of effectiveness. Though energy is a good indicator of whether or not significant damage will occur, it is more important to know the state that the energy is in. In this case energy in the form of velocity is undesired while energy in the form of elevation head has no negative impact. It has been estimated that roughly $70 \%$ of all damage to hydraulic structures is attributed to erosion through high-velocity water flow [7].

The baffled containment structure configuration with the extra two rows of baffles was determined to be a superior design for the following reasons. First, the flow downstream from the structure was much more uniform and did not have hydraulic jumps. Second, the velocity of the downstream flow was considerably lower. Lastly, this baffle containment structure performed similarly in both cross-Sections. This research could allow engineers to design more efficient and inexpensive low level outlet works for dams.

Another consideration that should be considered when constructing a concrete containment structure is a steel lining. Due to the fact that high velocities create considerable destruction and erosion a steel lining is advised. A steel lining is of utmost importance where the water jet exiting a Fixed-Cone valve strikes the walls and the baffles. These are the points where the direction of the water is most altered and water does not change direction easily. Steel liners also allow for any necessary repairs to occur with a fraction of the down time that would be required for concrete repairs. This is not to mention that repairs will not be necessary as often if a steel liner is used. Energy in the form of tail water elevation does not cause any damage unless the water elevation at a given point drops quite rapidly, not allowing the pressure in the underlying soil to equalize which can cause uplift of the concrete. This problem is more a function of downstream flow stability than it is of tail water depth. This is another reason that the baffle configuration with the extra two rows of baffles was the preferred design; because the hydraulic jump occurred over the last two rows of baffles the downstream flow pattern was stable and uniform. This design allows for more economical containment structures to be built because it allows for fewer engi- 
neering considerations and structures in the downstream channel (including riprap), it allows for smaller cross-Sections without dramatic changes in performance, and less maintenance reconstruction due to scour. In certain applications these reductions in cost could be considerable. With further interest and experimentation it would be possible to provide a design guide to those interested in designing more efficient Fixed-Cone valve containment structures.

\section{References}

[1] G. L. Beichley, "Hydraulic Model Studies of Scoggins Dam Fishtrap Aeration and Supply Structure," USBR REC-ERC-72-27, 1972.

[2] M. C. Johnson and R. Dham, "Innovative Energy-Dissipating Hood," Journal of Hydraulic Engineering, Vol. 138, No. 8, 2006, pp. 759-764.
doi:10.1061/(ASCE)0733-9429(2006)132:8(759)

[3] T. E. Helper and H. W. Peck, (1989). "Energy Dissipation Structure for Fixed-Cone Valves," Proceedings of the 1989 National Conference on Hydraulic Engineering, New Orleans, 14-18 August 1989, pp. 956-961.

[4] D. Colgate, "Hydraulic Model Studies of the Low-Level Outlet Works, LG-2 Development. Quebec, Canada," USBR REC-ERC-74-3, 1974.

[5] G. L. Beichley, "Hydraulic Model Studies of Portage Mountain Low Level Outlet Works," USBR Report No Hyd-508, 1966.

[6] G. L. Beichley, "Hydraulic model studies of an Energy Dissipator for a Fixed-Cone Valve at the Ute Dam Outlet Works," USBR REC-OCE-70-11, 1970.

[7] Y. Yin, D. Cui and X. Hu, "Study of Wear Performance of Hydraulic Concrete under High Speed Clear Water Jet," Chinese Science Abstracts, Vol. 6, No. 9, 2000, pp. 1181-1182. 